\title{
Eficácia da Arteterapia como tratamento complementar a depressão em idosos
}

\author{
Effectiveness of Art Therapy as a complementary treatment of depression in the elderly \\ Eficacia de la Arteterapia como tratamiento complementario a la depresión en el anciano
}

\begin{abstract}
Resumo
Objetivo: analisar a eficácia da arteterapia como um tratamento complementar ao farmacológico para idosos portadores da depressão, além de apresentar os sintomas da doença e a maneira como a mesma se manifesta nessa parcela da população. Metodologia: Trata-se de uma revisão integrativa de caráter exploratório e abordagem qualitativa, onde foram utilizadas fontes secundárias de pesquisa para o levantamento de dados bibliográficos. A busca bibliográfica foi realizada nas bases Lilacs, PubMed e Biblioteca Virtual em Saúde com corte temporal de 5 anos (2016-2021). Resultados: Foram analisados 2 estudos do tipo ensaio clínico e estudo observacional, nos quais idosos foram selecionados para fazerem parte de um grupo controle e um grupo experimental, onde passaram por testes que mediam o nível de depressão, ansiedade e autoestima, no começo do estudo e ao final da aplicação de sessões de arteterapia. Conclusão: Apesar das limitações e escassez de estudos relacionados ao tema, evidencia-se que a arteterapia demonstrou ser uma ótima forma de tratamento complementar a depressão, visto que com as sessões os idosos participantes puderam estabelecer relações interpessoais, combatendo um dos sintomas mais evidentes da doença, que é o isolamento social, além de terem apresentado uma melhora significativa nos sintomas da depressão, ansiedade e autoestima em relação ao grupo controle. Nesse contexto, é importante que novos estudos sejam realizados para que a prática seja mais difundida, visto que atualmente a arteterapia é demasiadamente subutilizada.
\end{abstract}

Palavras-chave: Arteterapia; Depressão; Idosos.

\begin{abstract}
Objective: Analyze the effectiveness of art therapy as a complementary treatment to the pharmacological for elderly people with depression, also present the disease symptons and the way it manifests itself in this portion of the population. Methodology: It is an integrative review of an exploratory nature and a qualitative approach, where secondary sources of research were used to collect bibliographic data. The bibliographic search was carried out in the Lilacs, PubMed and Virtual Health Library databases with a 5-year period (2016-2021). Results: Two studies of the clinical trial and observational study type were analyzed, in which the elderly were selected to be part of a control group and an experimental group, where they underwent tests that measured the level of depression, anxiety and self-esteem, at the beginning of the study and at the end of the application of art therapy sessions. Conclusion: Despite the limitations and scarcity of studies related to the theme, it is evident that art therapy has proved to be a great form of treatment complementary to depression, since with the sessions the elderly participants were able to establish interpersonal relationships, combating one of the most evident symptoms of depression disease, which is social isolation, in addition to showing a significant improvement in the symptoms of depression, anxiety and self-esteem in relation to the control group. In this context, it is important that further studies are carried out so that the practice is more widespread, since currently art therapy is too underused.
\end{abstract}


Keywords: Art therapy; Depression; Elderly.

\section{Resumen}

Objetivo: analizar la efectividad de la arteterapia como tratamiento complementario al tratamiento farmacológico en personas mayores con depresión, además de presentar los síntomas de la enfermedad y la forma en que se manifiesta en esta porción de la población. Metodología: Es una revisión integradora de carácter exploratorio y enfoque cualitativo, donde se utilizaron fuentes secundarias de investigación para la recolección de datos bibliográficos. La búsqueda bibliográfica se realizó en las bases de datos Lilacs, PubMed y Virtual Health Library con un lapso de 5 años (20162021). Resultados: Se analizaron dos estudios del tipo ensayo clínico y estudio observacional, en los cuales se seleccionaron ancianos para formar parte de un grupo control y un grupo experimental, donde se les realizaron pruebas que midieron el nivel de depresión, ansiedad y autoestima, al inicio del estudio y al final de la aplicación de las sesiones de arteterapia. Conclusión: A pesar de las limitaciones y escasez de estudios relacionados con la temática, es evidente que la arteterapia ha demostrado ser una gran forma de tratamiento complementario para la depresión, ya que con las sesiones los ancianos participantes lograron establecer relaciones interpersonales, combatiendo una de los síntomas más evidentes de la enfermedad depresiva, que es el aislamiento social, además de haber mejorado significativamente los síntomas de depresión, ansiedad y autoestima en relación al grupo control. En este contexto, es importante que se realicen nuevos estudios para que la práctica esté más extendida, ya que actualmente la arteterapia está demasiado infrautilizada.

Palabras clave: Terapia con arte; Depresión; Ancianos.

\section{Introdução}

A arte está presente na humanidade desde os primórdios de sua existência, sendo uma das muitas formas do ser humano se expressar quanto as suas percepções de mundo e sobre si mesmo, além de ser uma grande aliada da nossa sociedade atual, já que nos proporciona conhecimentos sobre os costumes e, até mesmo, a rotina de nossos antepassados, principalmente por meio das pinturas, objetos e instrumentos criados a partir de elementos da natureza.

O termo "Arteterapia" surgiu no final de 1940, sendo Adrian Hill uma das primeiras pessoas identificadas a utilizar este para definir uma técnica de criação de imagens como terapia no Reino Unido e, quase que ao mesmo tempo, Margaret Naumburg, nos Estados Unidos, também fez uso do termo para definir seu trabalho, utilizando como método a liberação do inconsciente por meio da expressão artística espontânea. Apesar de ambos fazerem uso do termo "arteterapia", suas abordagens possuíam certas diferenciações: Hill aplicava a arte como terapia, evidenciando a capacidade de cura inerente do processo criativo, enquanto Margaret aplicava a arte $n a$ terapia, expondo a importância da relação que se estabelecia entre o terapeuta, o paciente e a arte produzida (Edwards, 2014).

No Brasil, um nome que se destacou devido sua grande influência para a fundamentação teórica da arteterapia foi o de Nise da Silveira, que em 1946, no Centro Psiquiátrico Dom Pedro II, no Rio de Janeiro, desenvolveu um trabalho utilizando a arte para o tratamento de pessoas com transtornos mentais, tentando compreender as obras produzidas pelos seus pacientes e, assim, contribuindo de maneira significante para que se utilizassem métodos mais humanizado no tratamento destes (Nise: $\mathrm{O}$ Coração da Loucura, 2016).

De acordo com a Associação Americana de Arteterapia, a arteterapia pode ser definida como o uso terapêutico da atividade artística por pessoas que tenham vivenciado traumas e doenças como uma forma de ampliar o conhecimento sobre si mesmo, visando o aumento da autoestima e o aprendizado sobre como lidar melhor com sintomas e com tais experiências traumáticas, melhorando funções cognitivas, emocionais e motoras e estabelecendo uma relação de confiança e apoio entre o paciente e o profissional (American Art Therapy Association, 2017).

É notório que com o passar do tempo, devido à grande pressão social, ao estresse diário, a dificuldade em lidar com perdas, sejam elas quais forem, a falta de confiança em si e autoestima, além de fatores genéticos, são aspectos que favorecem de forma importante o aumento no índice de casos de depressão, que é considera atualmente como a "doença do século". Tal transtorno pode ser vivenciado por pessoas de diversas faixas etárias, porém é o transtorno mental que mais atinge a população idosa, de acordo com o Instituto Brasileiro de Geografia e Estatística (IBGE), apesar da grande dificuldade enfrentada pelos 
profissionais da saúde de se estabelecer um diagnóstico correto (SPDM - Associação Paulista para o Desenvolvimento da Medicina, 2016).

Com isso, vemos a importância de se ter uma equipe multidisciplinar para o auxílio do tratamento desses pacientes para que também seja garantido o princípio da integralidade da assistência formulado e garantido pelo SUS de acordo com a Lei $\mathrm{n}^{\circ}$ 8.080 de 1990. Segundo Wanda Horta, enfermeira formada pela USP em 1920, o ser humano possuí necessidades básicas que precisam ser atendidas para que se estabeleça um equilíbrio biopsicossocial e a enfermagem, como parte integrante da equipe de saúde, tem a função de prestar o cuidado de forma que as necessidades que estão em declínio possam ser recompostas (HORTA, 1960).

Portanto, o enfermeiro como parte da equipe multidisciplinar, deve orientar, auxiliar e estimular o paciente a recuperar suas necessidades básicas que são danificadas pelo transtorno depressivo, como a auto estima por exemplo, sempre de forma humanizada e com o intuito, também, de fazer com que esse indivíduo consiga se tornar independente dessa assistência através da prática do autocuidado, tendo a arteterapia como aliada ao tratamento no quesito de facilitar os desbloqueios psíquicos (HORTA, 1960).

Diante do exposto, emerge a seguinte questão norteadora: Quais são os benefícios da arte terapia para o tratamento da depressão na terceira idade e como identificar os sinais da doença nessa parcela da população? Tendo a utilização da mesma como complemento ao tratamento de depressão ao idoso como objeto de estudo.

O objetivo do presente artigo é analisar a eficácia e os impactos da arteterapia como um recurso complementar ao farmacológico para o tratamento da depressão no idoso e descrever os efeitos constatados da prática, além de facilitar o acesso à informação de como os sintomas da depressão podem ser observados na população idosa.

Dispondo da justificação de que realizando uma coletânea e fornecendo uma síntese de conteúdos de outros artigos encontrados, tendo em vista que tal agregação permita que as informações agrupadas neste ampliem o estudo e a visibilidade acerca do tema, contribuiremos para que a obtenção de conhecimento por parte da comunidade acadêmica se torne mais acessível e que dessa forma um atendimento mais humanizado para o tratamento de idosos nessas condições seja favorecido.

\section{Metodologia}

Trata-se de uma revisão integrativa de caráter exploratório e abordagem qualitativa realizado através de uma coleta de dados elaborada a partir de fontes secundárias de pesquisa.

Para Antonio Carlos Gil (2008), os tipos de pesquisa são classificados quanto aos seus objetivos e quanto aos seus procedimentos técnicos. Em particular, a pesquisa do tipo exploratória tem como objetivo proporcionar um estreitamento e maior proximidade com o tema abordado, tornando-o mais explícito. Além disso, Gil destaca que para que os dados da realidade se confrontem com a visão teórica é necessário estabelecer um delineamento, para que seja traçado um planejamento da coleta de dados relacionada a pesquisa.

Como delineamento, foi definido como procedimento técnico o levantamento de dados bibliográficos, que é elaborado a partir de materiais já publicados, principalmente livros e artigos científicos (GIL, 2008). Tal procedimento é de grande importância, sendo uma ótima forma de se iniciar um estudo, visto que o avanço da internet se tornou um grande meio de divulgação de artigos e pesquisas, além da constante atualização destes, possibilitando, assim, maior acesso ao conhecimento por parte da comunidade acadêmica.

Para o levantamento de dados, primeiramente foi realizada uma busca nas bases PubMed e LILACS (Literatura LatinoAmericana e do Caribe em Ciências da Saúde), seguida por uma busca mais ampla através do Portal Regional da Biblioteca Virtual em Saúde (BVS). Os critérios de escolha dos estudos foram: obras em português, inglês e espanhol publicadas entre 2016 a 2021 que continham as seguintes palavras-chave consideradas descritores no DeCs (Descritores em Ciências de Saúde) e MeSh 
(Medical Subject Headings) e suas combinações com os operadores booleanos: Arte Terapia, Depressão e Idosos, considerando que tais obras estivessem disponíveis para acesso completo.

Como critério de exclusão, foram descartados os artigos que não retratassem o tema Arteterapia como Tratamento Complementar a Depressão em Idosos como assunto principal e também aqueles que estivessem fora da temporalidade delimitada, bem como os que aparecessem em duplicidade nas bases, sendo considerados apenas uma vez.

$\mathrm{Na}$ busca, incialmente foram encontrados para uma leitura exploratória 20 artigos na base PubMed, 12 na Biblioteca Virtual em Saúde e apenas 1 na base de dados Lilacs. No total, obtivemos 28 artigos e 10 foram selecionados para a leitura integral. Após a leitura dos artigos, 3 foram selecionados para fazer parte da revisão, visto que respondiam aos critérios de inclusão citados anteriormente. No Quadro 1, estão descritas as etapas deste processo.

Quadro 1. Referências bibliográficas encontradas nas bases Lilacs, Pubmed e Biblioteca Virtual em Saúde a partir das palavras-chave selecionadas, Rio de Janeiro, RJ, Brasil, 2021.

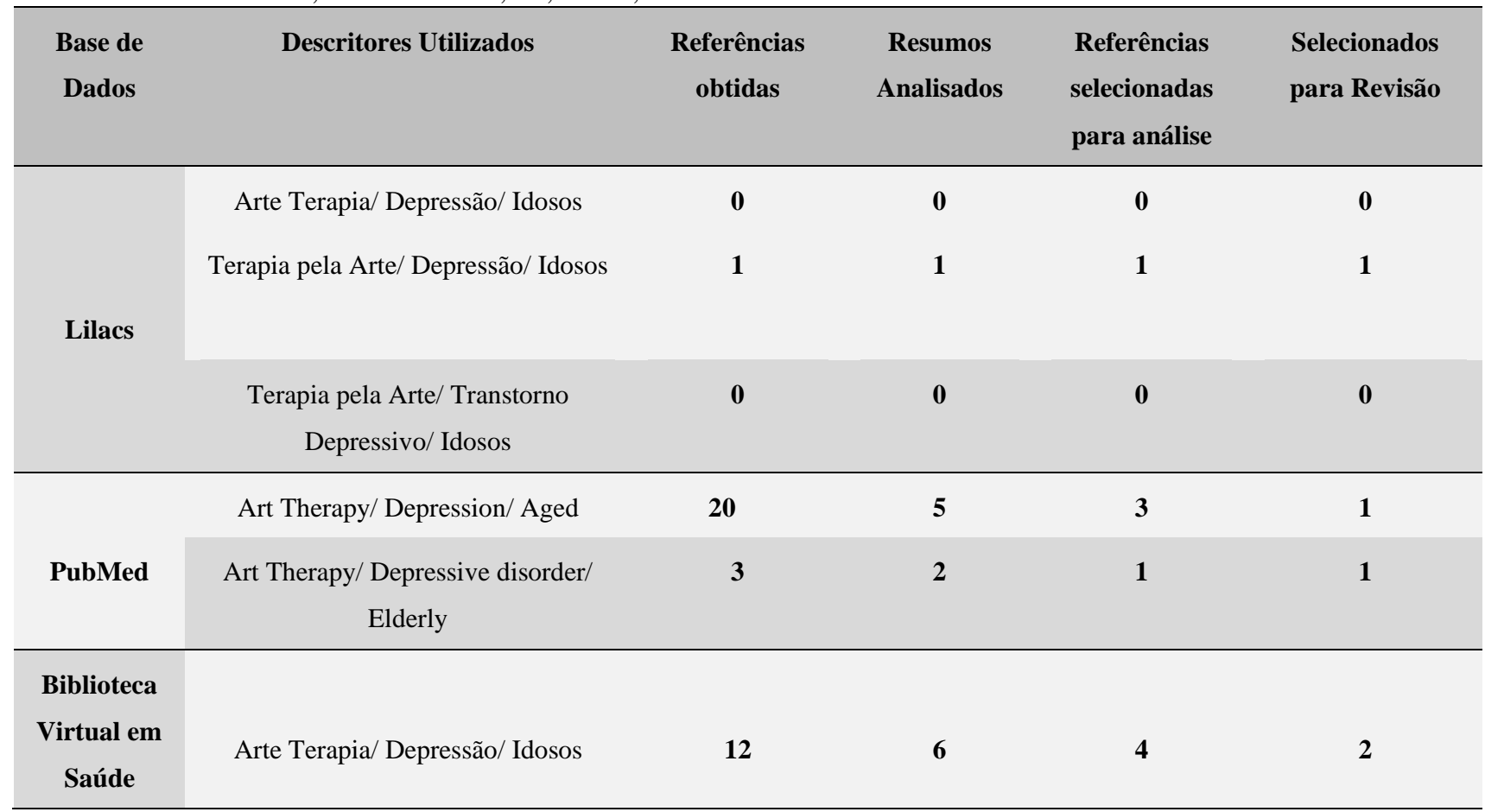

\section{Resultados}

Fonte: Autores.

Apresenta-se, no Quadro 2, os artigos que foram selecionados para a revisão, com o ano, país de publicação, seus autores, tipo de estudo, título e suas principais conclusões. 
Quadro 2. Referências selecionadas para a revisão de acordo com ano de publicação, país, autores, tipo de estudo e principais conclusões, Rio de Janeiro, RJ, Brasil, 2021.

\begin{tabular}{|c|c|c|c|c|c|}
\hline Ano & País & Autores & Tipo de Estudo & Título & Principais Conclusões \\
\hline 2017 & Brasil & Ciasca, EC. & $\begin{array}{l}\text { Ensaio clínico } \\
\text { controlado, } \\
\text { estudo } \\
\text { quantitativo }\end{array}$ & $\begin{array}{l}\text { Arteterapia e depressão: } \\
\text { efeitos da arteterapia como } \\
\text { terapia complementar no } \\
\text { tratamento da depressão } \\
\text { em idosos. }\end{array}$ & $\begin{array}{l}\text { O estudo mostra que as } \\
\text { idosas participantes do grupo } \\
\text { experimental apresentaram } \\
\text { redução nos sintomas } \\
\text { depressivos e ansiosos e } \\
\text { contribui para o } \\
\text { reconhecimento da } \\
\text { Arteterapia como um } \\
\text { complemento ao tratamento } \\
\text { farmacológico. }\end{array}$ \\
\hline 2018 & Brasil & $\begin{array}{l}\text { Ciasca, EC et } \\
\text { al. }\end{array}$ & $\begin{array}{l}\text { Ensaio clínico } \\
\text { controlado, } \\
\text { estudo } \\
\text { quantitativo }\end{array}$ & $\begin{array}{l}\text { Arteterapia como } \\
\text { tratamento complementar } \\
\text { a depressão em mulheres } \\
\text { idosas: um ensaio clínico } \\
\text { randomizado. }\end{array}$ & $\begin{array}{l}\text { As participantes do grupo } \\
\text { experimental apresentaram } \\
\text { melhora no mini exame do } \\
\text { estado mental e uma redução } \\
\text { nas pontuações de depressão } \\
\text { e ansiedade mais significativa } \\
\text { do que as participantes do } \\
\text { grupo controle. }\end{array}$ \\
\hline 2019 & Taiwan & $\begin{array}{l}\text { Yao Ching- } \\
\text { Teng, Yang } \\
\text { Ya-Ping e } \\
\text { Chen Yu-Chia }\end{array}$ & $\begin{array}{l}\text { Estudo } \\
\text { observacional, } \\
\text { qualitativo. }\end{array}$ & $\begin{array}{l}\text { Efeitos positivos da Arte } \\
\text { Terapia na depressão e } \\
\text { autoestima de idosos em } \\
\text { instituições de longa } \\
\text { permanência. }\end{array}$ & $\begin{array}{l}\text { As atividades artísticas } \\
\text { encorajaram os idosos } \\
\text { participantes a refletir sobre } \\
\text { diversos temas, propiciando } \\
\text { oportunidades de se } \\
\text { autoconhecerem e } \\
\text { expressarem seus } \\
\text { sentimentos, o que favoreceu } \\
\text { a diminuição dos sintomas } \\
\text { depressivos e o aumento da } \\
\text { autoestima. }\end{array}$ \\
\hline
\end{tabular}

Fonte: Autores.

Dos 3 artigos selecionados, 2 deles são ensaios clínicos controlados publicados pela mesma autora. Nesse estudo, há a participação de 56 mulheres idosas que estavam em tratamento medicamentoso já estável para a depressão. Houve uma separação das participantes em grupo controle (GC) e grupo experimental (GE), compostos respectivamente por 25 e 31 integrantes.

Foram utilizados, para avaliar o nível de depressão e ansiedade das participantes, a Escala de Depressão Geriátrica (GDS), que é um teste composto por 15 perguntas simples e de fácil entendimento amplamente utilizado para a avaliação e 
detecção de sintomas depressivos em pacientes idosos cuja pontuação maior ou igual a 5 é considerada um indicativo de depressão, a Escala de Depressão de Beck (BDI), teste com 21 perguntas que avaliam a gravidade dos sintomas depressivos em diversas faixas etárias e a Escala de Ansiedade de Beck (BAI), também com 21 questões, que avalia a intensidade dos sintomas de ansiedade, além de outros mecanismos para a análise de aspectos cognitivos e de qualidade de vida. Tanto a BDI quanto a BAI utilizam a pontuação maior ou igual a 12 como ponto de corte.

No início do estudo, o grupo controle apresentava uma pontuação média de 7,5 pontos na GDS, 15,9 na BDI e 13,7 na BAI, enquanto que no grupo experimental, as pontuações eram $\mathrm{GDS}=8,6, \mathrm{BDI}=20,9$ e BAI = 18,4. Não foi feita nenhuma intervenção psicológica nas participantes do grupo controle, já as integrantes do grupo experimental foram submetidas a 20 sessões de Arteterapia durante 20 semanas.

Ao final das sessões, as pontuações na GDS, BDI e BAI no grupo controle eram respectivamente 6,9, 14,4 e 10,8 e no grupo experimental eram 5,4, 12,3 e 9,5. Pode-se observar que em ambos os grupos houve uma diminuição nas pontuações, porém, no GE, é notável que ocorreu uma redução mais importante, o que evidencia uma melhora nos sintomas de depressão e ansiedade.

O terceiro artigo selecionado é um estudo observacional quase experimental, realizado em Taiwan com a participação de 55 idosos, sem distinção de sexo, que viviam em instituições de longa permanência. Os participantes também foram divididos em grupo controle, constituído por 26 idosos, e grupo experimental, com 29 integrantes. Ambos os grupos foram submetidos à Escala de Depressão Geriátrica, à Escala de Autoestima de Rosenberg (RSS) entre outros testes de cognição e capacidade física.

O grupo experimental, na fase pré teste, apresentou pontuação média de 8,55 na Escala de Depressão Geriátrica e 22,21 pontos na Escala de Autoestima de Rosenberg, já o grupo controle, no início do estudo, obteve pontuação média de 8,88 na GDS e 21,11 na RSS.

As atividades artísticas foram realizadas durante 12 semanas, e ao final do período de estudo, constatou-se as pontuações na GDS e na RSS 8,62 e 21,54, respectivamente, no grupo controle, enquanto que no grupo experimental as pontuações foram 2,90 na Escala de Depressão Geriátrica e 27,03 na Escala de Autoestima de Rosenberg, evidenciando que os sintomas de depressão diminuíram efetivamente e houve uma melhora na autoestima dos participantes do GE após as sessões de arteterapia.

\section{Discussão}

As sessões de arteterapia tinham em média uma duração de 90 a 100 minutos. No primeiro estudo, foram divididas em quatro fases: acolhimento, momento em que as idosas se preparavam para o início das atividades; relaxamento e imaginação dirigida, que é uma técnica de estimulação dos sentidos, onde há a indução de imagens positivas acompanhadas de som ambiente, que favorecem o relaxamento muscular e atenua os desconfortos ocasionados pelos transtornos de humor; produção artística, onde ocorriam de fato o início dos trabalhos artísticos e, por fim, a fase de compartilhamento, em que as participantes tinham a oportunidade de expressar seu sentimentos e reflexões que surgiram durante a atividade.

No segundo estudo, basicamente as mesmas etapas foram reproduzidas, com exceção da prática de relaxamento e imaginação dirigida. As fases foram: aquecimento, introdução do tema, criação da arte e, por fim, compartilhamento e exibição dos trabalhos.

Como foi retratado anteriormente, a depressão é um dos transtornos mentais que mais atinge a população idosa e, devido a dificuldade do diagnóstico correto nessa parcela da população e ao fato da maioria dos idosos negar tal condição, além de ela geralmente estar associada a outras patologias em comorbidade, a doença atinge um alto índice de mortalidade.

Para Simões (1966), os sintomas da depressão na terceira idade são relativamente inespecíficos como, por exemplo, a perda ou diminuição da força física, perturbações de sono, sentimentos frequentes de tristeza e ansiedade, perda de interesse nos prazeres habituais e isolamento social. 
O tratamento farmacológico para depressão em idosos deve ser conduzido de forma cuidadosa e gradativa devido à fragilidade e suscetibilidade desses pacientes apresentarem efeitos adversos, além da atenção à polifarmácia para que se evite interação medicamentosa que pode vir a intensificar tais efeitos, visto que a grande maioria faz uso de outros medicamentos para doenças já instaladas anteriormente.

Dessa forma, o tratamento da depressão na terceira idade em conjunto com psicoterapias é de grande valor, pois tais práticas representam uma escolha mais segura e sem efeitos adversos, além de que a combinação dos tratamentos se torna mais eficaz do que qualquer uma delas sozinhas (Cujipers, 2015).

\section{Conclusão}

Neste estudo, verificou-se que a arteterapia demonstrou ser de grande importância para a diminuição dos sintomas de depressão nos idosos, visto que ao final das sessões, os participantes dos estudos foram encorajados a expressar seus sentimentos e reflexões que foram vivenciados durante o processo, o que favoreceu uma melhora na interação social entre os participantes e o cultivo de um ambiente alegre, onde puderam manifestar suas criatividades de forma livre.

Ao final dos estudos, os pacientes que fizeram parte dos grupos experimentais, obtiveram uma melhora significativa nas pontuações das Escalas de Depressão Geriátrica, Beck Depressão, Beck Ansiedade e Autoestima de Rosenberg, evidenciando que o processo das oficinas de arteterapia diminuíram os sintomas da depressão e da ansiedade, além de melhorar a autoestima dos componentes dos grupos.

Denota-se que para este estudo foram encontradas algumas limitações como, por exemplo, a escassa quantidade de artigos que abordam o tema tendo a depressão por si só como problema principal e não decorrentes de uma outra condição.

Dessa forma, são necessários novos estudos, tanto qualitativos quanto quantitativos e intervencionais, para que seja gerado um embasamento teórico mais sólido e a eficácia da prática seja mais difundida com o propósito de fazer com que a arteterapia passe a ser prescrita de forma mais abrangente, visto que a mesma ainda é demasiadamente subutilizada.

\section{Referências}

Aguiar, A. P. \& Macri, R. (2010). Promovendo a qualidade de vida dos idosos através da arte terapia. RPCFO. https://doi.org/10.9789/2175-5361.2010.v0i0.\%p Brasil. (2015). Política nacional de práticas integrativas e complementares no SUS: Atitude de ampliação de acesso (2ª ed.), Brasília: Ministério da Saúde.

Ciasca, E. C., Ferreira, R. C., Santana, Carmen L. A., Forlenza, O. V., Santos, G. D., Brum, P. S. \& Nunes, P. V. (2018). Art therapy as an adjuvant treatment for depression in elderly women: a randomized controlled trial. Brazilian Journal of Psychiatry, 40(3), 256-63. https://doi.org/10.1590/1516-4446-2017-2250

Ciasca, E. C. (2017). Arteterapia e depressão: efeitos da arteterapia como terapia complementar no tratamento da depressão em idosos. Dissertação de Mestrado, Faculdade de Medicina, Universidade de São Paulo. Doi: 10.11606/D.5.2017.tde-06062017-082522

Ching-Teng Y., Ya-Ping Y., \& Yu-Chia C. (2019). Positive effects of art therapy on depression and self-esteem of older adults in nursing homes. Tandfonline, Taiwan. https://doi.org/10.1080/00981389.2018.1564108

Coqueiro, N. F., (2010). Arte terapia como dispositivo terapêutico em saúde mental. Acta paulista de enfermagem, São Paulo

Cuijpers, P., Smit, F. P., Vikram, D., Amit. L., Juan, R., \& Charles, F., (2015). Prevention of depressive disorders in older adults. PsyCh Journal, EUA. https://doi.org/10.1002/pchj.86

Edwards, D., (2016). Art Therapy: Creative Therapies in Practice. United Kingdom. SAGE.

Ferrari, J., F. \& Dalacorte, R. R. (2017). Uso da Escala de Depressão Geriátrica de Yesavage para avaliar a prevalência de depressão em idosos hospitalizados. PUCRS.17 (1), 3-8.

Freitas, A. Z. V. M., Vasconcelos, A. K. M., Silva, R. O., Silva, S. P. C. S., Piagge, C. S. L. D. \& Cavalcanti, Y. W. (2020) A arteterapia influencia na qualidade de vida da pessoa idosa? Revisão sistemática. Research, Society and Development, 9(11) 2525-3409. http://dx.doi.org/10.33448/rsd-v9i11.10329

Gato, J. M., Zenevicz, L. T., Madureira, V. S. F., Silva, T. G., Celich, K. L. S., Souza, S. S. \& Léo, M. M. F. (2018). Saúde mental e qualidade de vida de pessoas idosas. Avances en Enfermería, 36(3), 302-310. https://doi.org/10.15446/av.enferm.v36n3.68498

Gil, A. C., (2008). Como elaborar projetos de pesquisa. 4. Ed. São Paulo: Atlas. 
Research, Society and Development, v. 10, n. 7, e14010716411, 2021

(CC BY 4.0) | ISSN 2525-3409 | DOI: http://dx.doi.org/10.33448/rsd-v10i7.16411

Horta, W. A. (1974). Enfermagem: Teoria, conceitos, princípios e processo. Revista da Escola de Enfermagem da USP, 8(1), 7 17. https://dx.doi.org/10.1590/0080-6234197400800100007

Júnior, G. S., Okuno, M. F. P., Oliveira, L. M., Barbosa, D. A., Alonso, A. C., Fram, D. S. \& Belasco, A. G. S. (2019). Qualidade de vida de idosos institucionalizados com e sem sinais de depressão. Revista Brasileira de Enfermagem, 72(2) 127-133. http://dx.doi.org/10.1590/0034-7167-2018-0316

Martins, R. M., (2008). A depressão no idoso. Instituto Politécnico de Viseu, Portugal. http://hdl.handle.net/10400.19/364

Ramos, F. P., Silva, S. C., Freitas, D. F., GangussuL. M. B., Bicalho, A. H., Sousa, B. V. O., Rametta, Z. M. J., Rametta, F. J., Rametta, L. P. M., Nascimento, C. I. C., Santos, S. H. S., \& Guimarães, T. A. (2019). Fatores associados à depressão em idoso. Revista Eletrônica Acervo Saúde, https://doi.org/10.25248/reas.e239.2019

Silva, B. C. M., et. al. (2021) Importância da identificação do diagnóstico de enfermagem ao paciente com depressão senil na atenção básica. Research, Society and Development, 10(2) 2525-3409. http://dx.doi.org/10.33448/rsd-v10i2.12770

Souza, M. T., Silva, M. D., \& Carvalho, R. (2010). Revisão integrativa: o que é e como fazer. Einstein (São Paulo), 8(1), 102106. https://doi.org/10.1590/s1679-45082010rw1134

Stella, F., Gobbi, S., Corazza, D. I., \& Costa, J. L. R., (2002). Depressão no Idoso: Diagnóstico, Tratamento e Benefícios da Atividade Física, Universidade Estadual Paulista, São Paulo. https://doi.org/10.5016/6473

Tier, C. G., Santos, S. S. C., Pelzer, M. T. \& Bulhosa, M. S (2007). Escalas de avaliação da depressão em idosos. RI FURG.

http://repositorio.furg.br/handle/1/4404 